\title{
Necesidades nutricionales e hídricas en el tenis
}

\author{
Jesús Sanchez, Fernando Mata, Moises Grimaldi y Raul Domínguez \\ Universidad Pablo de Olavide de Sevilla, España
}

RESUMEN

El tenis es un deporte con altos requerimientos nutricionales y de líquidos. Un adecuado abordaje dietético-nutricional asegura el estado de salud y el rendimiento óptimo del tenista. Todo abordaje debe fundamentarse en la determinación de aquellos factores que disminuyen el rendimiento deportivo para, posteriormente, fijar una serie de objetivos en base a dichos factores $y$, por último, establecer las estrategias más adecuadas para conseguir cada uno de los objetivos propuestos.
Palabras clave: necesidades, nutrición, hidratación, rendimiento, tenis.

Recibido: 17 Feb 2017

Aceptado: $20 \mathrm{Jul} 2017$

Autor correspondiente: Jesús Sanchez, Universidad Pablo de Olavide de Sevilla, España. Correo electrónico: asanchez@upo.es

\section{INTRODUCCIÓN}

El rendimiento y la salud de un deportista está íntimamente ligado a una adecuada nutrición (Kondric, Sekulic, Uljevic, Gabrilo, \& Zvan, 2013), siendo de gran relevancia en la prevención y recuperación de lesiones (Moran et al., 2012).

Un tenista ha de conjugar altos niveles de fuerza y potencia muscular, velocidad y agilidad, coordinación y toma de decisiones en condiciones de fatiga y estrés mental durante largos períodos de tiempo (lacoboni, 2001), ya que un partido de tenis suele tener una duración de 90 minutos, pudiendo alargarse hasta 4-5 horas (Kondric et al., 2013). Además, un punto en tenis tiene una duración media de 7-10 segundos, con periodos de recuperación entre 10-90 segundos, según se cambie de lado de la pista o no (O'Donoghue \& Ingram, 2001).

Proporcionar una adecuada alimentación que ayude a controlar los factores limitantes del rendimiento, que facilite una buena recuperación tras los entrenamientos y partidos, y que ayude a crear unas mejores adaptaciones fisiológicas en pro del rendimiento del tenista se presta de vital importancia.

\section{FACTORES LIMITANTES DEL RENDIMIENTO EN TENIS}

La identificación de los factores limitantes del rendimiento en el tenis y el establecimiento de objetivos nutricionales que tengan en cuenta dichos factores limitantes son fundamentales (Maughan, 2003).

La bajada en el rendimiento debida a la incapacidad de mantener un adecuado control motor o de producir fuerza, producto de la fatiga y las demandas fisiológicas del tenis, tiene como principales factores limitantes los reflejados en la figura 1.

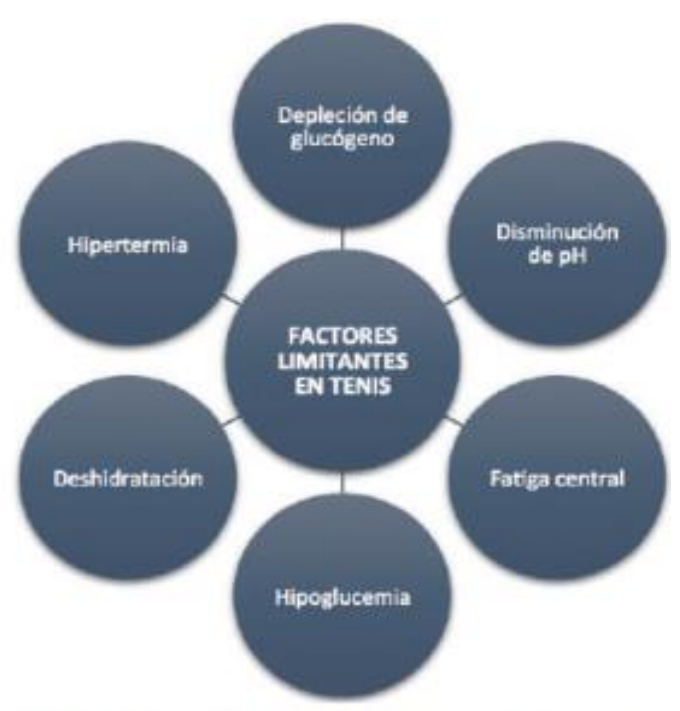

Figura 1. Factores limitantes del rendimiento en tenis. 


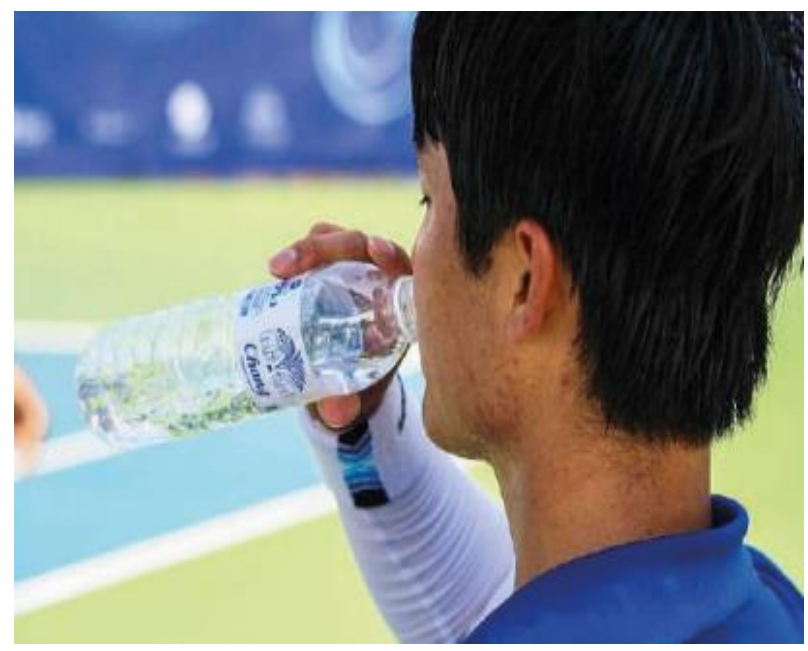

Las reservas de glucógeno muscular y hepático pueden deplecionarse en partidos de alta exigencia o entrenamientos de gran volumen (Ferrauti, Pluim, Busch, \& Weber, 2003), afectando al rendimiento por incapacidad energética (Hornery, Farrow, Mujika, \& Young, 2007). Junto a ésta puede acontecer una disminución de glucemia, que disminuye el rendimiento físico y mental, acompañándose de procesos catabólicos (Kovacs, 2008). Además, una disminución del pH durante el ejercicio puede dar lugar a una disminución de la obtención de energía a través del sistema de los fosfágenos y de la contracción muscular (Wallimann, Tokarska-Schlattner, \& Schlattner, 2011), y un aumento de la percepción subjetiva del esfuerzo (Price \& Moss, 2007). Todo esto puede dar lugar al uso de aminoácidos de cadena ramificada como sustrato energético en lo que se denomina fatiga central (Blomstrand, 2006).

La pérdida de líquido corporal es otro de los factores limitantes en tenis. Ésta disminuye la capacidad termorreguladora del organismo (Binkley, Beckett, Casa, Kleiner, \& Plummer, 2002), el gasto cardíaco (González-Alonso, Mora-Rodríguez, \& Coyle, 2000), e incrementa la glucolisis anaeróbica (Ranchordas, Rogersion, Ruddock, Killer, \& Winter, 2013) y la aparición de calambres (Sawka et al., 2007). La hipertermia se correlaciona con la duración de los partidos (Morante \& Brotherhood, 2008) y con el grado de deshidratación (GonzálezAlonso et al., 2000), pudiendo tener consecuencias vitales.

\section{NECESIDADES ENERGÉTICAS}

Mantener un determinado peso corporal durante toda la temporada es uno de los retos en tenis, habida cuenta de la gran variabilidad en las demandas energéticas en función de la fase de entrenamiento en la que se encuentre o del paso en sucesivas rondas de una competición (Ranchordas et al., 2013).

La alimentación además de satisfacer las necesidades diarias en cuanto a requerimientos de vitaminas y minerales, deberá responder a las demandas del tenista en cuanto a aporte de macronutrientes y momento de ingesta, de manera que sirva para reforzar su estado de salud y rendimiento deportivo (Tavío \& Domínguez Herrera, 2014).

\section{Hidratos de Carbono (HC)}

La reposición de las reservas de glucógeno es el objetivo principal en la ingestión de $\mathrm{HC}$; siendo también importantes para evitar el sobreentrenamiento y el correcto funcionamiento del sistema inmunitario. La disminución de la resistencia está íntimamente ligada a la reducción de las reservas de glucógeno (Domínguez, 2012), produciendo un aumento de citoquinas y cortisol (Nieman, Zwetsloot, Lomiwes, Meaney, \& Hurst, 2016). Para paliar esto, y en función del tiempo de recuperación entre esfuerzos y de la intensidad y duración de los mismos, un tenista necesita de 6-10 g/ kg/día (Ranchordas et al., 2013). Esta ingesta de $\mathrm{HC}$ ha de realizarse antes, durante y después en función de lo anteriormente dicho (figura 2).

Los HC de bajo índice glucémico son fundamentales en la ingesta previa, asegurando una estabilidad en la glucemia durante el mismo y siendo recomendable al menos 2 horas antes (Fernández, Miranda, \& Jiménez, 2008), ya que se ha visto una disminución del rendimiento al ingerir alimentos con alto índice glucémico en los 45 minutos previos (Sousa et al., 2010).

La oxidación del glucógeno, la estabilidad de la glucemia y la economía en las reservas de glucógeno se verá favorecida al ingerir HC durante el ejercicio (Ostojic \& Mazic, 2002). La percepción subjetiva del esfuerzo y la respuesta del cortisol fue menor en un partido de tenis en el que se realizó una ingesta de $0,5 \mathrm{~g} / \mathrm{kg} / \mathrm{hora}$ de $\mathrm{HC}$, además de mantener la glucemia estable (Gomes et al., 2013). Recientemente se ha señalado que la ingesta de $\mathrm{HC}$ durante el esfuerzo físico puede elevarse hasta los $90 \mathrm{~g} / \mathrm{h}$, siempre que se respete la relación glucosafructosa de 2:1 (Jeukendrup, 2013), superándose la recomendación clásica de 60gr/h (Sawka et al., 2007).

\section{ANTES: átima ingesta rica en $\mathrm{CH}$ de bajo indice gucémico y con 2 h de antelación}

\section{DURANTE: hasta $90 \mathrm{~g} / \mathrm{h}$ manteniendo la relación glucosa-fiuctosa $2: 1$}

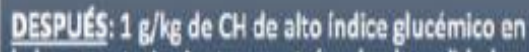
la hora posterior junto a proteina de alita calidad.

Figura 2. Recomendaciones de $\mathrm{CH}$ en el tenis. 
La capacidad de síntesis de glucógeno es mayor en los 30-60 minutos posteriores al esfuerzo (Domínguez, 2012), recomendándose que durante la hora posterior se ingieran $\mathrm{HC}$ de alto índice glucémico a razón de $1 \mathrm{~g} / \mathrm{kg}$. Algunos autores proponen simultanear dicha ingesta con proteínas para potenciar el mencionado efecto, recomendando que la proteína que acompañe los HC sea de alto valor (Moore et al., 2008).

\section{Proteínas (PRO)}

Aunque las PRO solamente serán utilizados con fines energéticos al darse una disminución del glucógeno y un incremento del cortisol, éstas son fundamentales para el buen funcionamiento del organismo (Aparicio, Nebot, Heredia, \& Aranda, 2010). Debido a que los tenistas presentan un alto porcentaje de masa magra y por su posible contribución al metabolismo energético, las necesidades se establecen entre $1,6 \mathrm{~g} / \mathrm{kg} /$ día (Ranchordas et al., 2013) y 1,8 g/ kg/día (Phillips $\&$ Van Loon, 2011). Además, habrá que tener en cuenta el momento de ingestión y la calidad de la fuente proteica (Ranchordas et al., 2013; Suárez López, Kizlansky, \& López, 2006). La ingestión simultánea de $\mathrm{CH}$ y PRO tras el ejercicio es fundamental en la recuperación y el mantenimiento de la masa magra (Stark, Lukaszuk, Prawitz, \& Salacinski, 2012), recomendándose $6 \mathrm{~g}$ de aminoácidos esenciales, equivalentes a $20 \mathrm{~g}$ de PRO de alto valor biológico (Borsheim et al., 2004), o $0,3 \mathrm{~g} / \mathrm{kg}$ de PRO de alto valor biológico, dado que valores por encima no serán utilizadas para la síntesis de nuevas PRO (Moore et al., 2008).

\section{Lípidos (LIP)}

Aunque no existe un requerimiento específico para la ingesta de LIP en tenis, estos son de gran importancia, ya que, difícilmente se puede llegar a los requerimientos mínimos de vitaminas liposolubles y ácidos grasos esenciales si no se consumen (Robertson, Benardot, \& Mountjoy, 2014). Además, los triglicéridos intramusculares son una fuente importante de energía en ejercicios de larga duración, jugando un importante papel en los periodos de recuperación en tenis (Horvath, Eagen, Ryer-Calvin, \& Pendergast, 2000). Teniendo en cuenta el gasto energético diario y las demandas en HC y PRO, las calorías diarias en forma de ácidos grasos deberán representar entre 20\%-35\% del total, primando la ingesta de ácidos grasos poliinsaturados versus saturados (Mozaffarian, Micha, \& Wallace, 2010) y estableciéndose como límite de ingesta de LIP los $2 \mathrm{~g} / \mathrm{kg}$ / día en tenis (Ranchordas et al., 2013).

\section{NECESIDADES HÍDRICAS}

La deshidratación es uno de los principales factores limitantes en tenis. La reducción de la masa corporal por deshidratación durante la práctica deportiva no debe ser superior al 1,5-2\%
(Sawka et al., 2007), por lo que implantar un plan de hidratación es importante en entrenamiento y competición (figura 3). Tenistas con altas tasas de sudoración pueden llegar a perder entre 2,3-2,7\% kg/h (Bergeron, 2003); para evitar esto, Kovacs (2008) propone que un tenista debe beber $250 \mathrm{ml} / \mathrm{h}$ durante la práctica.

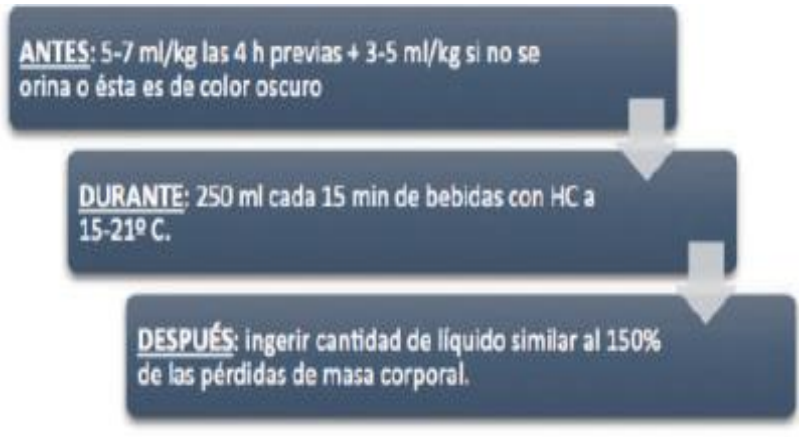

Figura 3. Necesidades hídricas en el tenis.

Además, para garantizar un correcto estado de hidratación, el tenista debería ingerir $5-7 \mathrm{ml} / \mathrm{kg}$ en las 4 horas previas, que se complementaran, en el caso de que durante dicho periodo no se orine o la micción sea densa u oscura con 3-5 ml/kg más (Sawka et al., 2007). La ingesta de líquido tras el ejercicio supondrá $150 \%$ de la perdida producida durante el mismo, teniendo en cuenta que la bebida a una temperatura de entre 150 y 210 C y con HC estimulará la sed (Sawka et al., 2007).

\section{CONCLUSIÓN}

La nutrición puede tener un impacto importante en el tenis, jugando un papel fundamental en el mantenimiento de la salud de los tenistas y afectando positivamente en el rendimiento de los mismos. La adopción de estrategias nutricionales puede ayudar a mejorar la tolerancia al ejercicio y a recuperar mejor tras los entrenamientos y la competición.

\section{REFERENCES}

Aparicio, V. A., Nebot, E., Heredia, J. M., \& Aranda, P. (2010). Efectos metabólicos, renales y óseos de las dietas hiperproteicas. Papel regulador del ejercicio. Revista Andaluza de Medicina Del Deporte, 3(4), 153-158.

Bergeron, M. F. (2003). Heat cramps: fluid and electrolyte challenges during tennis in the heat. Journal of Science and Medicine in Sport, 6(1), 19-27. Retrieved from http://www.ncbi.nlm.nih. gov/pubmed/12801207. https://doi.org/10.1016/S14402440(03)80005-1

Binkley, H. M., Beckett, J., Casa, D. J., Kleiner, D. M., \& Plummer, P. E. (2002). National Athletic Trainers' Association Position Statement: Exertional Heat Illnesses. Journal of Athletic Training, 37(3), 329-343. Retrieved from http://www.ncbi.nlm.nih.gov/ pubmed/12937591 
Blomstrand, E. (2006). A role for branched-chain amino acids in reducing central fatigue. The Journal of Nutrition, 136(2), 544S-547S. Retrieved from http://www.ncbi.nlm.nih.gov/ pubmed/16424144. https://doi.org/10.1093/jn/136.2.544S

Borsheim, E., Cree, M. G., Tipton, K. D., Elliott, T. A., Aarsland, A., \& Wolfe, R. R. (2004). Effect of carbohydrate intake on net muscle protein synthesis during recovery from resistance exercise. Journal of Applied Physiology, 96(2), 674-678. https://doi.org/10.1152/japplphysiol.00333.2003

Domínguez, R. (2012). Necesidades de hidratos de carbono en el deportista de resistencia. Motricidad Humana, 13, 51-56. Retrieved from http://www.revistamotricidad.com/?rmh_ articulos $=$ necesidades-de-hidratos-de-carbono-eneldeportista-de-resistencia. https://doi.org/10.5027/jmh-Vol13Issue1(2012)art52

Fernández, J. M., Miranda, J. L., \& Jiménez, F. P. (2008). Índice glucémico y ejercicio físico. Revista Andaluza de Medicina Del Deporte, 1(3), $116-124$.

Ferrauti, A., Pluim, B. M., Busch, T., \& Weber, K. (2003). Blood glucose responses and incidence of hypoglycaemia in elite tennis under practice and tournament conditions. Journal of Science and Medicine in Sport, 6(1), 28-39. https://doi.org/10.1016/S14402440(03)80006-3

Gomes, R., Capitani, C., Ugrinowitsch, C., Zourdos, M., FernandezFernandez, J., Mendez-Villanueva, A., \& Aoki, M. (2013). Does carbohydrate supplementation enhance tennis match play performance? Journal of the International Society of Sports Nutrition, 10(1), 46. https://doi.org/10.1186/1550-2783$\underline{10-46}$

González-Alonso, J., Mora-Rodríguez, R., \& Coyle, E. F. (2000). Stroke volume during exercise: interaction of environment and hydration. American Journal of Physiology. Heart and Circulatory Physiology, 278(2), H321-30. Retrieved from http://www.ncbi. nlm.nih.gov/pubmed/10666060.

https://doi.org/10.1152/ajpheart.2000.278.2.H321

Hornery, D. J., Farrow, D., Mujika, I., \& Young, W. B. (2007). Caffeine, carbohydrate, and cooling use during prolonged simulated tennis. International Journal of Sports Physiology and Performance, 2(4), 423-38. Retrieved from http://www.ncbi. nlm.nih.gov/pubmed/19171960. https://doi.org/10.1123/ijspp.2.4.423

Horvath, P. J., Eagen, C. K., Ryer-Calvin, S. D., \& Pendergast, D. R. (2000). The effects of varying dietary fat on the nutrient intake in male and female runners. Journal of the American College of Nutrition, 19(1), 42-51. Retrieved from http://www.ncbi.nlm. nih.gov/pubmed/10682875.

https://doi.org/10.1080/07315724.2000.10718914 https://doi.org/10.1080/07315724.2000.10718913

lacoboni, M. (2001). Playing tennis with the cerebellum. Nature Neuroscience, 4(6), 555-556. https://doi.org/10.1038/88365. https://doi.org/10.1038/88365

Jeukendrup, A. E. (2013). Multiple transportable carbohydrates and their benefits. Sports Science Exchange, 26(108), 1-5.
Kondric, M., Sekulic, D., Uljevic, O., Gabrilo, G., \& Zvan, M. (2013). Sport nutrition and doping in tennis: an analysis of athletes' attitudes and knowledge. Journal of Sports Science \& Medicine, 12(2), 290-7. Retrieved from http://www.ncbi.nlm.nih.gov/pubmed/24149808

Kovacs, M. S. (2008). A Review of Fluid and Hydratio in Competitive Tennis. International Journal of Sports Physiology and Performance, 3(4), 413-423. https://doi.org/10.1123/ijspp.3.4.413

Maughan, R. J. (2003). Nutritional status, metabolic responses to exercise and implications for performance. Biochemical Society $\left.\begin{array}{lll}\text { Transactions, } & 31(\mathrm{Pt} & 6\end{array}\right)$, https://doi.org/10.1042/bst0311267

Moore, D. R., Robinson, M. J., Fry, J. L., Tang, J. E., Glover, E. I., Wilkinson, S. B., ... Phillips, S. M. (2008). Ingested protein dose response of muscle and albumin protein synthesis after resistance exercise in young men. American Journal of Clinical Nutrition, 89(1), 161168. https://doi.org/10.3945/ajen.2008.26401

Moran, D. S., Heled, Y., Arbel, Y., Israeli, E., Finestone, A., Evans, R. K., \& Yanovich, R. (2012). Dietary intake and stress fractures among elite male combat recruits. Journal of the International Society of Sports Nutrition, 9(1), 6. https://doi.org/10.1186/1550-2783-9-6

Morante, S. M., \& Brotherhood, J. R. (2008). Thermoregulatory responses during competitive singles tennis. British Journal of Sports Medicine, 42(9), 736-741. https://doi.org/10.1136/bjsm.2007.037002

Mozaffarian, D., Micha, R., \& Wallace, S. (2010). Effects on coronary heart disease of increasing polyunsaturated fat in place of saturated fat: a systematic review and meta-analysis of randomized controlled trials. PLoS Medicine, 7(3), e1000252. https://doi.org/10.1371/journal.pmed.1000252

Nieman, D. C., Zwetsloot, K. A., Lomiwes, D. D., Meaney, M. P., \& Hurst, R. D. (2016). Muscle Glycogen Depletion Following $75-\mathrm{km}$ of Cycling Is Not Linked to Increased Muscle IL-6, IL-8, and MCP-1 mRNA Expression and Protein Content. Frontiers in Physiology, 7, 431. https://doi.org/10.3389/fphys.2016.00431

O'Donoghue, P., \& Ingram, B. (2001). A notational analysis of elite tennis strategy. Journal of Sports Sciences, 19(2), 107-115. https://doi.org/10.1080/026404101300036299

Ostojic, S. M., \& Mazic, S. (2002). Effects of a carbohydrateelectrolyte rink on specific soccer tests and performance. Journal of Sports Science \& Medicine, 1(2), 47-53. Retrieved from http://www.ncbi.nlm.nih.gov/pubmed/24688270

Phillips, S. M., \& Van Loon, L. J. C. (2011). Dietary protein for athletes: From requirements to optimum adaptation. Journal of Sports Sciences, 29(sup1), S29-S38. https://doi.org/10.1080/02640414.2011.619204

Price, M., \& Moss, P. (2007). The effects of work:rest duration on physiological and perceptual responses during intermittent exercise and performance. Journal of Sports Sciences, 25(14), 1613-1621. https://doi.org/10.1080/02640410701287248 
Ranchordas, M. K., Rogersion, D., Ruddock, A., Killer, S. C., \& Winter, E. M. (2013). Nutrition for tennis: practical recommendations. Journal of Sports Science \& Medicine, 12(2), 211-24. Retrieved from http://www.ncbi.nlm.nih.gov/pubmed/24149799

Robertson, S., Benardot, D., \& Mountjoy, M. (2014). Nutritional Recommendations for Synchronized Swimming. International Journal of Sport Nutrition and Exercise Metabolism, 24(4), 404413. https://doi.org/10.1123/ijsnem.2014-0013

Sawka, M. N., Burke, L. M., Eichner, E. R., Maughan, R. J., Montain, S. J., \& Stachenfeld, N. S. (2007). American College of Sports Medicine position stand. Exercise and fluid replacement. Medicine and Science in Sports and Exercise, 39(2), 377-90.

Stark, M., Lukaszuk, J., Prawitz, A., \& Salacinski, A. (2012). Protein timing and its effects on muscular hypertrophy and strength in individuals engaged in weight-training. Journal of the International Society of Sports Nutrition, 9(1), 54. https://doi.org/10.1186/1550-2783-9-54
Sousa, M. V. de, Altimari, L. R., Okano, A. H., Coelho, C. F., Altimari, J. M. Teixeira, O., ... Cyrino, E. S. (2010). Pre-exercise high concentration carbohydrate supplementation impairs the performance on high intensity cycling exercise. Revista Andaluza de Medicina Del Deporte (España) Num.4 Vol.3.

Suárez López, M. M., Kizlansky, A., \& López, L. B. (2006). Evaluación de la calidad de las proteínas en los alimentos calculando el escore de aminoácidos corregido por digestibilidad. Nutrición Hospitalaria, 21(1), 47-51.

Tavío, P., \& Domínguez Herrera, R. (2014). Necesidades dietéticonutricionales en la práctica profesional del tenis: una revisión. Nutrición Clínica Y Dietética Hospitalaria, ISSN 02116057, Vol. 34, No. 2, 2014, Págs. 18-28, 34(2), 18-28.

Wallimann, T., Tokarska-Schlattner, M., \& Schlattner, U. (2011). The creatine kinase system and pleiotropic effects of creatine. Amino Acids, 40(5), 1271-1296. https://doi.org/10.1007/s00726-011$\underline{0877-3}$

Derechos de Autor (c) 2017 Jesús Sanchez, Fernando Mata, Moises Grimaldi y Raul Domínguez.

\section{ITF Academy}

Este texto está protegido por una licencia CreativeCommons 4.0.

Usted es libre para Compartir -copiar y redistribuir el material en cualquier medio o formato- y Adaptar el documento -remezclar, transformar y crear a partir del material- para cualquier propósito, , incluso para fines comerciales, siempre que cumpla la condición de:

Atribución: Usted debe dar crédito a la obra original de manera adecuada, proporcionar un enlace a la licencia, e indicar si se han realizado cambios. Puede hacerlo en cualquier forma razonable, pero no de forma tal que sugiera que tiene el apoyo del licenciante o lo recibe por el uso que hace de la obra.

Resumendelicencia - Textocompletodelalicencia 